\title{
Quantum Yang-Baxter Equation, Braided Semigroups, and Dynamical Yang-Baxter Maps
}

\author{
Diogo Kendy MATSUMOTO and Youichi SHIBUKAWA \\ Waseda University and Hokkaido University \\ Dedicated to Professor Kimio Ueno on the occasion of his sixtieth birthday
}

\begin{abstract}
We prove that braided semigroups with suitable conditions can produce solutions to the quantum Yang-Baxter equation in every tensor category. As an application, some dynamical Yang-Baxter maps, set-theoretic solutions to a version of the quantum dynamical Yang-Baxter equation, are constructed.
\end{abstract}

\section{Introduction}

The quantum Yang-Baxter equation (QYBE for short) has attracted much interest in mathematics and physics since the pioneering works [2, 31]. R-matrices, matrix solutions to this equation, gave the quantum groups $[4,11,30]$. We can similarly define dynamical quantum groups $[6,8]$ by means of solutions to the quantum dynamical Yang-Baxter equation (QDYBE for short) [10], a generalization of the QYBE.

Drinfel'd [5] suggested to study set-theoretical solutions [7, 9, 19, 20] to the QYBE, which are called Yang-Baxter maps [29]. This Yang-Baxter map plays an important role in defining discrete integrable systems [1]. On the other hand, dynamical Yang-Baxter maps $[17,21,22,23]$ are set-theoretical solutions to a version of the QDYBE. This dynamical Yang-Baxter map yields bialgebroids [25] and discrete integrable systems through 3D compatible ternary systems [14]. Furthermore, suitable homogeneous pre-systems [12], related to reductive homogeneous spaces, can produce the dynamical Yang-Baxter map.

Now we introduce simple examples of the Yang-Baxter map ([3], [22, Remark 6.2 (2)]). Let $G$ be a group, and let $e_{G}$ denote its unit element. The maps $\sigma_{1}$ and $\sigma_{2}$ on $G \times G$ defined by

$$
\sigma_{1}(a, b)=\left(e_{G}, a b\right) \text { and } \sigma_{2}(a, b)=\left(a b, e_{G}\right) \quad(a, b \in G)
$$

satisfy the QYBE

$$
\sigma_{i} \times \operatorname{id}_{G} \circ \operatorname{id}_{G} \times \sigma_{i} \circ \sigma_{i} \times \operatorname{id}_{G}=\operatorname{id}_{G} \times \sigma_{i} \circ \sigma_{i} \times \operatorname{id}_{G} \circ \operatorname{id}_{G} \times \sigma_{i} \quad(i=1,2) .
$$

Received December 19, 2013; revised May 2, 2014

Mathematics Subject Classification: Primary 16T25; Secondary 18D10, 20M50, 20N05

Key words and phrases: Braided semigroups, Dynamical Yang-Baxter maps, Left quasigroups, Quantum YangBaxter equations 
The group $G$ can produce Yang-Baxter maps.

These pairs $\left(G, \sigma_{i}\right)(i=1,2)$ are not always braided groups [24, 28], which are useful in constructing the Yang-Baxter map. However, they still satisfy several properties in the definition of the braided group.

The aim of this paper is to generalize the examples (1.1) from the viewpoint of category theory; braided semigroups play a role of the above pairs $\left(G, \sigma_{i}\right)(i=1,2)$ in this generalization. As an application, we construct dynamical Yang-Baxter maps by means of left quasigroups $[18,26]$.

The organization of this paper is as follows. Section 2 presents a brief summary of a tensor category Set $_{H}$ before constructing dynamical Yang-Baxter maps, which generalize (1.1). In Section 3, our main results are stated and proved. Braided semigroups with suitable conditions give birth to morphisms satisfying the QYBE in every tensor category. In Section 4 , we construct the braided semigroups in the previous section from semigroups with a left or right unit. The final section deals with a proof of Theorem 1 about the construction of dynamical Yang-Baxter maps, by making use of the results in Sections 3 and 4.

\section{Tensor category $\mathrm{Set}_{H}$ and dynamical Yang-Baxter maps}

In this section, we construct dynamical Yang-Baxter maps, which generalize (1.1), after a brief summary of a tensor category $\operatorname{Set}_{H}[23,24]$. For category theory, see [13, 15].

Let $H$ be a non-empty set. We denote by Set $_{H}$ the following category: its object is a pair $\left(X, \cdot_{X}\right)$ of a set $X$ and a map $\cdot X: H \times X \ni(\lambda, x) \mapsto \lambda \cdot X x \in H$; its morphism $f:(X, \cdot X) \rightarrow(Y, \cdot Y)$ is a map $f: H \rightarrow \operatorname{Map}(X, Y)$ satisfying that $\lambda \cdot Y f(\lambda)(x)=$ $\lambda \cdot X X \quad(\forall \lambda \in H, \forall x \in X)$; the identity 1 and the composition $\circ$ are defined by

$$
1_{X}(\lambda)(x)=x \quad(\lambda \in H, x \in X) \quad \text { and } \quad(g \circ f)(\lambda)=g(\lambda) \circ f(\lambda) \quad(\lambda \in H)
$$

for objects $X, Y, Z$ and morphisms $f: X \rightarrow Y, g: Y \rightarrow Z$. We will often write $\lambda \cdot X x$ simply $\lambda x$.

This Set $_{H}$ is a tensor category. In fact, the tensor product $X \otimes Y$ of the objects $X=$ $(X, \cdot X)$ and $Y=(Y, \cdot Y)$ is a pair $(X \times Y, \cdot)$ of the Cartesian product $X \times Y$ and the following map $: H \times(X \times Y) \rightarrow H$.

$$
\lambda \cdot(x, y)=(\lambda \cdot X x) \cdot Y \quad y \quad(\lambda \in H,(x, y) \in X \times Y) .
$$

The tensor product of the morphisms $f: X \rightarrow X^{\prime}$ and $g: Y \rightarrow Y^{\prime}$ is defined by $(f \otimes g)(\lambda)(x, y)=(f(\lambda)(x), g(\lambda x)(y))(\lambda \in H,(x, y) \in X \times Y)$. The definitions of the associativity constraint $a$, the unit $I$, and the left and the right unit constraints $l, r$ are as follows: $a_{X Y Z}(\lambda)((x, y), z)=(x,(y, z)) ; I=(\{e\}, \cdot I)$, a pair of the set $\{e\}$ of one element and the map $\cdot I$ defined by $\lambda \cdot{ }_{I} e=\lambda ; l_{X}(\lambda)(e, x)=x=r_{X}(\lambda)(x, e)$.

Definition 1. A morphism $\sigma: X \otimes X \rightarrow X \otimes X$ of Set $_{H}$ is a dynamical Yang-Baxter map [17, 21, 23, 24], iff $\sigma$ satisfies the QYBE (2.1) in Set $_{H}$.

$a \circ \sigma \otimes 1_{X} \circ a^{-1} \circ 1_{X} \otimes \sigma \circ a \circ \sigma \otimes 1_{X}=1_{X} \otimes \sigma \circ a \circ \sigma \otimes 1_{X} \circ a^{-1} \circ 1_{X} \otimes \sigma \circ a$. 
Here, $a=a_{X, X, X}$.

If this dynamical Yang-Baxter map is an automorphism in $\mathbf{S e t}_{H}$, then it is exactly a Yang-Baxter operator [13, Definition XIII.3.1].

REMARK 1. (1) The dynamical Yang-Baxter map in the above definition is called a dynamical braiding map satisfying an invariance condition [22, Definition 2.8].

(2) If $H$ is a set of one element, then the tensor category $\operatorname{Set}_{H}$ is the tensor category Set consisting of sets, and the dynamical Yang-Baxter map is exactly a Yang-Baxter map.

Every left quasigroup $[18,26]$ can produce dynamical Yang-Baxter maps.

DEFINITION 2. A left quasigroup $Q$ is a non-empty set, together with a binary operation (.) on $Q$ such that the left translation map $L(a): Q \ni b \mapsto a \cdot b \in Q$ is bijective for all $a \in Q$.

For simplicity of notation, we write $a b(a, b \in Q)$ instead of $a \cdot b$, and denote $L(a)^{-1}(b)(\in Q)$ by $a \backslash b$. Here, $L(a)^{-1}: Q \rightarrow Q$ is the inverse of $L(a)$. We note that the binary operation of the left quasigroup is not always associative.

EXAMPLE 1. (1) The group $G$ is a left quasigroup.

(2) The set $\mathbb{Z}$ of integers, together with a binary operation $a \cdot b:=b-a(a, b \in \mathbb{Z})$, is a left quasigroup. This binary operation is not associative.

(3) Let $V$ denote the vector space $(\mathbb{Z} / 3 \mathbb{Z})^{4}$ over the finite field $\mathbb{Z} / 3 \mathbb{Z}$. We define the binary operation (.) on $V$ by

$$
a \cdot b=\left(a_{1}+b_{1}, a_{2}+b_{2}, a_{3}+b_{3}, a_{4}+b_{4}+\left(a_{3}-b_{3}\right)\left(a_{1} b_{2}-a_{2} b_{1}\right)\right)
$$

for $a=\left(a_{1}, a_{2}, a_{3}, a_{4}\right), b=\left(b_{1}, b_{2}, b_{3}, b_{4}\right) \in V$. The pair $(V, \cdot)$ is a left quasigroup; moreover, this is a smallest commutative Moufang loop that is not a group [18, Example IV.5.1].

For a left quasigroup $Q$ and $\lambda \in Q$, we define the binary operation $(\cdot \lambda)$ on $Q$ by

$$
a \cdot \lambda b=\lambda \backslash((\lambda a) b) \quad(a, b \in Q) .
$$

We denote by $Q_{\lambda}$ the set $Q$ with the above binary operation $(\cdot \lambda)$ (2.2).

PROPOSITION 1. $Q_{\lambda}$ is a left quasigroup for any $\lambda \in Q$.

REMARK 2 . The binary operation $(\cdot \lambda)$ is called a left derivative of $(\cdot)$ with respect to $\lambda \in Q[18$, Section III.5].

A relation $\sim$ on $Q$ defined by

$$
\lambda \sim \mu \Leftrightarrow a \cdot \lambda b=a \cdot{ }_{\mu} b \quad(\forall a, b \in Q)
$$

is an equivalence relation on $Q$. We write $H:=Q / \sim$.

Proposition 2. If $\lambda \sim \mu$, then $\lambda a \sim \mu a$ for any $a \in Q$. 
PROOF. By the definition (2.2), $a \cdot \lambda(x \cdot \lambda a y)=(a \cdot \lambda x) \cdot \lambda y$ for $x, y \in Q$, and consequently $a \cdot \lambda(x \cdot \lambda a y)=a \cdot \mu(x \cdot \mu a y)=a \cdot \lambda(x \cdot \mu a y)$, since $\lambda \sim \mu$. On account of Proposition 1, $x \cdot \lambda_{a} y=x \cdot \mu a y$ for $x, y \in Q$, which is the desired result.

REMARK 3. Each equivalence class $[\lambda] \in H$ containing $\lambda \in Q$ is the set $\lambda \cdot N_{l}\left(Q_{\lambda}\right)$. Here, $N_{l}\left(Q_{\lambda}\right)$ is a left nucleus [18, Definition I.3.2] of the left quasigroup $Q_{\lambda}: N_{l}\left(Q_{\lambda}\right)=$ $\left\{a \in Q_{\lambda} \mid(a \cdot \lambda x) \cdot \lambda y=a \cdot \lambda(x \cdot \lambda y) \quad\left(\forall x, y \in Q_{\lambda}\right)\right\}$.

We define a map (.) $: H \times Q \rightarrow H$ by $[\lambda] \cdot a:=[\lambda a](\lambda, a \in Q)$. Because of Proposition 2 , this definition makes sense.

Proposition 3. $Q=(Q, \cdot)$ is an object of Set $_{H}$.

Let $s: H \rightarrow Q$ be a right inverse of the projection $Q \ni \lambda \mapsto[\lambda] \in H$; that is, $s: H \rightarrow Q$ is a map satisfying $s([\lambda]) \sim \lambda$ for all $\lambda \in Q$. We denote by $\sigma_{1}([\lambda])$ and $\sigma_{2}([\lambda])$ $(\lambda \in Q)$ the maps on $Q \times Q$ defined by:

$$
\begin{aligned}
& \sigma_{1}([\lambda])(a, b)=(s([\lambda]) \backslash s([\lambda]), \lambda \backslash((\lambda a) b)) ; \\
& \sigma_{2}([\lambda])(a, b)=(\lambda \backslash((\lambda a) b), s([(\lambda a) b]) \backslash s([(\lambda a) b])) \quad(a, b \in Q) .
\end{aligned}
$$

THEOREM 1. Both $\sigma_{1}$ and $\sigma_{2}$ are dynamical Yang-Baxter maps.

We will give a proof of this theorem in Section 5 after clarifying the structure of $\sigma_{i}$ $(i=1,2)$ in Sections 3 and 4 from the viewpoint of category theory.

EXAMPLE 2. (1) If the left quasigroup $Q$ is a group $G$ (See Example 1 (1)), then the set $H$ has only one element, and the maps $\sigma_{i}:=\sigma_{i}([\lambda])(i=1,2, \lambda \in G)$ are the same as those in (1.1) for any right inverse $s$ (See also Remark 1 (2)).

(2) If the left quasigroup $Q$ is $\mathbb{Z}$ in Example 1 (2), then $\lambda \sim \mu \Leftrightarrow \lambda=\mu(\lambda, \mu \in \mathbb{Z})$. As a result, the set $H$ is isomorphic to $\mathbb{Z}$ as sets, and every right inverse $s$ satisfies $s([\lambda])=\lambda(\lambda \in \mathbb{Z})$. The maps $\sigma_{i}([\lambda])(i=1,2, \lambda \in \mathbb{Z})$ are as follows:

$$
\begin{aligned}
& \sigma_{1}([\lambda])(a, b)=(2 \lambda, b-a+2 \lambda) ; \\
& \sigma_{2}([\lambda])(a, b)=(b-a+2 \lambda, 2 b-2 a+2 \lambda) \quad(\lambda, a, b \in \mathbb{Z}) .
\end{aligned}
$$

(3) If the left quasigroup $Q$ is $V$ in Example 1 (3), then $1<\#(H)<\#(V)(=81)$. The element $1_{V}:=(0,0,0,0)$ is the unit element of $(V, \cdot)$, and the inverse $a^{-1}$ of $a(\in V)$ is $-a$. Because $(V, \cdot)$ is a Moufang loop, $\left(b a^{-1}\right) a=b$ for any $a, b \in V$ [18, Section IV.1]. By virtue of Proposition 2,

$$
a \sim b \Rightarrow a a^{-1} \sim b a^{-1} \Leftrightarrow 1_{V} \sim b a^{-1} \Rightarrow 1_{V} a \sim\left(b a^{-1}\right) a \Leftrightarrow a \sim b ;
$$

that is, $a \sim b \Leftrightarrow b a^{-1} \sim 1_{V}$. A straightforward computation shows that $a \sim 1_{V} \Leftrightarrow$ $a=\left(0,0,0, a_{4}\right)\left(a_{4} \in \mathbb{Z} / 3 \mathbb{Z}\right)$, and the relation $a \sim b$ is consequently equivalent to that $b=a+(0,0,0, r)(\exists r \in \mathbb{Z} / 3 \mathbb{Z}) . H(=V / \sim)$ is thus a set of order 27. Since the orders of the sets $H$ and $V$ are different, the method in [22] does not produce 
this example directly. Finally, the maps $\sigma_{i}([\lambda])(i=1,2, \lambda \in V)$ for any right inverse $s$ are as follows:

$$
\sigma_{1}([\lambda])(a, b)=\left(1_{V}, x\right) ; \sigma_{2}([\lambda])(a, b)=\left(x, 1_{V}\right) \quad(\lambda, a, b \in V) .
$$

Here, the element $x \in V$ is defined by

$$
\begin{aligned}
& x=\left(a_{1}+b_{1}, a_{2}+b_{2}, a_{3}+b_{3},\right. \\
& \left.\qquad a_{4}+b_{4}+\lambda_{1}\left(a_{2} b_{3}-a_{3} b_{2}\right)+\lambda_{2}\left(a_{3} b_{1}-a_{1} b_{3}\right)+\left(\lambda_{3}+a_{3}-b_{3}\right)\left(a_{1} b_{2}-a_{2} b_{1}\right)\right) \\
& \text { for } \lambda=\left(\lambda_{1}, \lambda_{2}, \lambda_{3}, \lambda_{4}\right), a=\left(a_{1}, a_{2}, a_{3}, a_{4}\right), b=\left(b_{1}, b_{2}, b_{3}, b_{4}\right) \in V .
\end{aligned}
$$

\section{QYBE and braided semigroups}

This section establishes a relation between the QYBE and braided semigroups in tensor categories, which play an essential role in the proof of Theorem 1.

Let $C=(C, \otimes, a, I, l, r)$ be a tensor category. That is to say, $C$ is a category with a tensor product $\otimes: C \times C \rightarrow C$, an associativity constraint $a: \otimes \circ(\otimes \times \mathrm{id}) \rightarrow \otimes \circ(\mathrm{id} \times \otimes)$, a unit object $I$, and left and right unit constraints $l: \otimes(I \times \mathrm{id}) \rightarrow \mathrm{id}, r: \otimes(\mathrm{id} \times I) \rightarrow$ id with respect to $I$, satisfying the pentagon axiom and the triangle axiom. We denote by $1_{X}: X \rightarrow X$ the identity morphism of an object $X$.

A pair $\left(X, m_{X}\right)$ of an object $X$ and a morphism $m_{X}: X \otimes X \rightarrow X$ is a semigroup, iff $m_{X}$ satisfies

$$
m_{X} \circ\left(m_{X} \otimes 1_{X}\right)=m_{X} \circ\left(1_{X} \otimes m_{X}\right) \circ a_{X, X, X} .
$$

This morphism $m_{X}$ is called a multiplication. A pair $\left(X, \Delta_{X}\right)$ of an object $X$ and a morphism $\Delta_{X}: X \rightarrow X \otimes X$ is a co-semigroup, the dual concept of the semigroup, iff $\Delta_{X}$ satisfies

$$
a_{X, X, X} \circ\left(\Delta_{X} \otimes 1_{X}\right) \circ \Delta_{X}=\left(1_{X} \otimes \Delta_{X}\right) \circ \Delta_{X} .
$$

The morphism $\Delta_{X}$ is said to be a comultiplication.

Let $\sigma_{X Y}: X \otimes Y \rightarrow Y \otimes X$ be a morphism of the tensor category $C$.

Definition 3. A matched pair of semigroups $X=\left(X, m_{X}\right)$ and $Y=\left(Y, m_{Y}\right)$ (Cf. $[16,24,27,28])$ is a triple $\left(X, Y, \sigma_{X Y}\right)$ satisfying:

$$
\begin{aligned}
& \left(1_{Y} \otimes m_{X}\right) \circ a_{Y, X, X} \circ\left(\sigma_{X Y} \otimes 1_{X}\right) \circ a_{X, Y, X}^{-1} \circ\left(1_{X} \otimes \sigma_{X Y}\right) \\
= & \sigma_{X Y} \circ\left(m_{X} \otimes 1_{Y}\right) \circ a_{X, X, Y}^{-1} ; \\
& \left(m_{Y} \otimes 1_{X}\right) \circ a_{Y, Y, X}^{-1} \circ\left(1_{Y} \otimes \sigma_{X Y}\right) \circ a_{Y, X, Y} \circ\left(\sigma_{X Y} \otimes 1_{Y}\right) \\
= & \sigma_{X Y} \circ\left(1_{X} \otimes m_{Y}\right) \circ a_{X, Y, Y} .
\end{aligned}
$$

A pair $\left(X, \sigma_{X}\right)$ of a semigroup $X$ and a morphism $\sigma_{X}: X \otimes X \rightarrow X \otimes X$ is called a braided semigroup, iff the triple $\left(X, X, \sigma_{X}\right)$ is a matched pair of semigroups. 
REMARK 4. The matched pair $\left(X, Y, \sigma_{X Y}\right)$ of semigroups gives birth to a semigroup. In fact, $\left(Y \otimes X, m_{Y \otimes X}\right)$ is a semigroup with the morphism $m_{Y \otimes X}:(Y \otimes X) \otimes(Y \otimes X) \rightarrow Y \otimes X$ defined by

$$
m_{Y \otimes X}=m_{Y} \otimes m_{X} \circ a_{Y \otimes Y, X, X} \circ\left(a_{Y, Y, X}^{-1} \circ 1_{Y} \otimes \sigma_{X Y} \circ a_{Y, X, Y}\right) \otimes 1_{X} \circ a_{Y \otimes X, Y, X}^{-1} .
$$

$\left(X \otimes X, m_{X \otimes X}\right)$ is hence a semigroup, if $\left(X, \sigma_{X}\right)$ is a braided semigroup.

Let $X=\left(X, m_{X}\right)$ be a semigroup with a comultiplication $\Delta_{X}: X \rightarrow X \otimes X$. We write $\sigma:=\Delta_{X} \circ m_{X}: X \otimes X \rightarrow X \otimes X$.

THEOREM 2. If the pair $(X, \sigma)$ is a braided semigroup, then $\sigma$ satisfies the QYBE (2.1) in the tensor category $C$.

Proof. Because $\sigma=\Delta_{X} \circ m_{X}$,

$($ Right-hand-side of $(2.1))=\left(1_{X} \otimes \Delta_{X}\right) \circ\left(1_{X} \otimes m_{X}\right) \circ a \circ\left(\sigma \otimes 1_{X}\right) \circ a^{-1} \circ\left(1_{X} \otimes \sigma\right) \circ a$.

Here, $a=a_{X, X, X}$. On account of (3.3), the right-hand-side of the above equation is $\left(1_{X} \otimes \Delta_{X}\right) \circ \sigma \circ\left(m_{X} \otimes 1_{X}\right)$. By making use of $\sigma=\Delta_{X} \circ m_{X}$ again, $\left(1_{X} \otimes \Delta_{X}\right) \circ \sigma \circ\left(m_{X} \otimes 1_{X}\right)=$ $\left(1_{X} \otimes \Delta_{X}\right) \circ \Delta_{X} \circ m_{X} \circ\left(m_{X} \otimes 1_{X}\right)$. From (3.4), a similar argument induces that

(Left-hand-side of (2.1)) $=a \circ\left(\Delta_{X} \otimes 1_{X}\right) \circ \Delta_{X} \circ m_{X} \circ\left(1_{X} \otimes m_{X}\right) \circ a$.

This completes the proof in view of (3.1) and (3.2).

REMARK 5. (1) The dual concept of the braided semigroup is a braided cosemigroup. Let $\sigma_{X Y}: X \otimes Y \rightarrow Y \otimes X$ be a morphism of a tensor category $C$. A matched pair of co-semigroups $\left(X, \Delta_{X}\right)$ and $\left(Y, \Delta_{Y}\right)$ is a triple $\left(X, Y, \sigma_{X Y}\right)$ satisfying:

$\sigma_{X Y} \otimes 1_{X} \circ a_{X, Y, X}^{-1} \circ 1_{X} \otimes \sigma_{X Y} \circ a_{X, X, Y} \circ \Delta_{X} \otimes 1_{Y}=a_{Y, X, X}^{-1} \circ 1_{Y} \otimes \Delta_{X} \circ \sigma_{X Y} ;$ $1_{Y} \otimes \sigma_{X Y} \circ a_{Y, X, Y} \circ \sigma_{X Y} \otimes 1_{Y} \circ a_{X, Y, Y}^{-1} \circ 1_{X} \otimes \Delta_{Y}=a_{Y, Y, X} \circ \Delta_{Y} \otimes 1_{X} \circ \sigma_{X Y}$.

A pair $\left(X, \sigma_{X}\right)$ of a co-semigroup $X$ and a morphism $\sigma_{X}: X \otimes X \rightarrow X \otimes X$ is a braided co-semigroup, iff the triple $\left(X, X, \sigma_{X}\right)$ is a matched pair of co-semigroups.

(2) The matched pair $\left(X, Y, \sigma_{X Y}\right)$ of co-semigroups defines a co-semigroup $(X \otimes$ $\left.Y, \Delta_{X \otimes Y}\right)$. Here,

$$
\Delta_{X \otimes Y}:=a_{X \otimes Y, X, Y} \circ\left(a_{X, Y, X}^{-1} \circ 1_{X} \otimes \sigma_{X Y} \circ a_{X, X, Y}\right) \otimes 1_{Y} \circ a_{X \otimes X, Y, Y}^{-1} \circ \Delta_{X} \otimes \Delta_{Y} .
$$

From this fact, $\left(X \otimes X, \Delta_{X \otimes X}\right)$ is a co-semigroup, if $\left(X, \sigma_{X}\right)$ is a braided cosemigroup.

(3) A dual of Theorem 2 is also true. Let $\left(X, \Delta_{X}\right)$ be a co-semigroup with a multiplication $m_{X}$. We set $\sigma:=\Delta_{X} \circ m_{X}$. If $(X, \sigma)$ is a braided co-semigroup, then $\sigma$ satisfies the QYBE. The proof is similar to that of Theorem 2. 


\section{Semigroups with left or right unit}

In this section, we construct the braided semigroups in Theorem 2 by means of semigroups with a left or right unit.

Let $C=(C, \otimes, a, I, l, r)$ be a tensor category, $S$ an object of $C$, and $\eta: I \rightarrow S$ a morphism of $C$. We define the morphisms $\Delta_{i}: S \rightarrow S \otimes S(i=1,2)$ by

$$
\Delta_{1}=\left(\eta \otimes 1_{S}\right) \circ l_{S}^{-1} \quad \text { and } \quad \Delta_{2}=\left(1_{S} \otimes \eta\right) \circ r_{S}^{-1} .
$$

Proposition 4. Both $\left(S, \Delta_{1}\right)$ and $\left(S, \Delta_{2}\right)$ are co-semigroups.

Proof. From (4.1), $\Delta_{1} \circ \eta=\eta \otimes \eta \circ l_{I}^{-1}=\eta \otimes \eta \circ r_{I}^{-1}=\Delta_{2} \circ \eta$, which induces

$$
\Delta_{1} \otimes 1_{S} \circ \Delta_{1}=\Delta_{2} \otimes 1_{S} \circ \Delta_{1} \quad \text { and } 1_{S} \otimes \Delta_{2} \circ \Delta_{2}=1_{S} \otimes \Delta_{1} \circ \Delta_{2} .
$$

By virtue of the triangle axiom,

$$
a_{S, S, S} \circ \Delta_{2} \otimes 1_{S}=1_{S} \otimes \Delta_{1} .
$$

It follows immediately from (4.2) and (4.3) that $\Delta_{1}$ and $\Delta_{2}$ satisfy (3.2). This proves the proposition.

A morphism $\eta: I \rightarrow S$ is called a left unit (resp. a right unit) of a semigroup $\left(S, m_{S}\right)$, iff $\eta$ satisfies $m_{S} \circ \eta \otimes 1_{S}=l_{S}\left(\right.$ resp. $m_{S} \circ 1_{S} \otimes \eta=r_{S}$ ).

Let $\left(S, m_{S}\right)$ be a semigroup with a left or right unit $\eta$. With the aid of the above proposition, the morphisms $\Delta_{1}$ and $\Delta_{2}$ are comultiplications of $S$. We define the morphisms $\sigma_{i}$ $(i=1,2)$ by $\sigma_{i}:=\Delta_{i} \circ m_{S}$.

PROPOSITION 5. For $i=1,2,\left(S, \sigma_{i}\right)$ is a braided semigroup.

PROOF. The following lemma and (3.1) immediately establish (3.3) and (3.4) for the case $i=1$.

LEMMA 1. $\Delta_{1}$ satisfies:

$$
\begin{aligned}
& 1_{S} \otimes m_{S} \circ a_{S, S, S} \circ \Delta_{1} \otimes 1_{S}=\Delta_{1} \circ m_{S} ; \\
& m_{S} \circ m_{S} \otimes 1_{S} \circ a_{S, S, S}^{-1} \circ 1_{S} \otimes \Delta_{1}=m_{S} ; \\
& m_{S} \otimes 1_{S} \circ a_{S, S, S}^{-1} \circ 1_{S} \otimes \Delta_{1} \circ \Delta_{1}=\Delta_{1} .
\end{aligned}
$$

For the proof of the case $i=2$, we use:

$$
\begin{aligned}
& m_{S} \otimes 1_{S} \circ a_{S, S, S}^{-1} \circ 1_{S} \otimes \Delta_{2}=\Delta_{2} \circ m_{S} ; \\
& m_{S} \circ 1_{S} \otimes m_{S} \circ a_{S, S, S} \circ \Delta_{2} \otimes 1_{S}=m_{S} ; \\
& 1_{S} \otimes m_{S} \circ a_{S, S, S} \circ \Delta_{2} \otimes 1_{S} \circ \Delta_{2}=\Delta_{2} .
\end{aligned}
$$

This completes the proof. 
Proof of LEMma 1. The naturality of the left unit constraint $l$, together with the fact that $l_{S \otimes S} \circ a_{I, S, S}=l_{S} \otimes 1_{S}$, implies (4.4).

If $\eta$ is a left unit of the semigroup $\left(S, m_{S}\right)$, then

$$
m_{S} \circ \Delta_{1}=1_{S},
$$

which induces (4.5) by virtue of the associativity (3.1) of $m_{S}$.

If $\eta$ is a right unit of $\left(S, m_{S}\right)$, then

$$
m_{S} \otimes 1_{S} \circ a_{S, S, S}^{-1} \circ 1_{S} \otimes \Delta_{1}=1_{S \otimes S} .
$$

In fact, the left-hand-side of (4.11) is $\left(m_{S} \circ 1_{S} \otimes \eta\right) \otimes 1_{S} \circ a_{S, I, S}^{-1} \circ 1_{S} \otimes l_{S}^{-1}$. The triangle axiom, together with the fact that $\eta$ is a right unit, gives (4.11), which consequently yields (4.5).

The proof of (4.6) is immediate by making (3.2), (4.10), and (4.11) into account.

Some $\sigma_{i}$ are idempotent.

PROPOSITION 6. If $\eta$ is a left unit, then $\sigma_{1}^{2}=\sigma_{1}$; and, if $\eta$ is a right unit, then $\sigma_{2}^{2}=$ $\sigma_{2}$.

Proof. From (4.10) and the fact that $\sigma_{1}=\Delta_{1} \circ m_{S}, \sigma_{1}^{2}=\sigma_{1}$, if $\eta$ is a left unit. A similar argument induces that $\sigma_{2}^{2}=\sigma_{2}$, if $\eta$ is a right unit.

REMARK 6. (1) $\left(S, \sigma_{i}\right)(i=1,2)$ in Proposition 5 are also braided co-semigroups because of (3.2) and (4.4)-(4.9). As a result, $S \otimes S$ is a semigroup with respect to $m_{S \otimes S}$, but also a co-semigroup with respect to $\left(\Delta_{i}\right)_{S \otimes S}$. Here, $m_{S \otimes S}$ is the morphism (3.5) for $X=Y=S$, and $\left(\Delta_{i}\right)_{S \otimes S}$ is the morphism (3.6) for $X=Y=S$ and $\Delta_{X}=\Delta_{Y}=\Delta_{i}$.

(2) The quartet $\left(S, m_{S}, \Delta_{i}, \sigma_{i}\right)(i=1,2)$ is a "bi-semigroup." In fact, it follows from (4.4)-(4.9) that

$$
m_{S \otimes S} \circ \Delta_{i} \otimes \Delta_{i}=\Delta_{i} \circ m_{S} .
$$

The morphism $\Delta_{i}: S \rightarrow S \otimes S$ hence respects the semigroup structures (See (1)). On the other hand, from (3.5) and (3.6), (4.12) is exactly the same as

$$
m_{S} \otimes m_{S} \circ\left(\Delta_{i}\right)_{S \otimes S}=\Delta_{i} \circ m_{S},
$$

which means that $m_{S}: S \otimes S \rightarrow S$ respects the co-semigroup structures. Therefore, we can regard $S$ as a bi-semigroup.

\section{Proof of Theorem 1}

This section is devoted to a proof of Theorem 1. We follow the notation used in Section 2. 
Let $Q=(Q, \cdot)$ be a left quasigroup (See Definition 2). We denote by $H$ the set of all equivalence classes of the relation (2.3) on $Q$.

For any $[\lambda] \in H(\lambda \in Q), m_{Q}([\lambda])$ is the map from $Q \times Q$ to $Q$ defined by $m_{Q}([\lambda])(a, b)=a \cdot \lambda b$ for $a, b \in Q$ (Cf. [23, (3.3)]). For $(\cdot \lambda)$, see (2.2). On account of (2.3), this definition is unambiguous.

Proposition 7. $m_{Q}: Q \otimes Q \rightarrow Q$ is a morphism of Set $_{H}$. Moreover, $Q=$ $\left(Q, m_{Q}\right)$ is a semigroup in $\operatorname{Set}_{H}$.

Let $s: H \rightarrow Q$ be a right inverse of the projection $Q \ni \lambda \mapsto[\lambda] \in H$. For any $[\lambda] \in H$ $(\lambda \in Q), \eta_{Q}^{(s)}([\lambda])$ is the map from $I=\{e\}$ to $Q$ defined by $\eta_{Q}^{(s)}([\lambda])(e)=s([\lambda]) \backslash s([\lambda])$.

Proposition 8. (1) $\eta_{Q}^{(s)}: I \rightarrow Q$ is a morphism of Set $_{H}$.

(2) $\eta_{Q}^{(s)}$ is a left unit of the semigroup $\left(Q, m_{Q}\right)(C f .[23,(3.5)])$.

Proof. We give only the proof of (1). For the proof, it is sufficient to show that $[\lambda] \eta_{Q}^{(s)}([\lambda])(e)=[\lambda] e(\lambda \in Q, I=\{e\})$. Because $s([\lambda]) \sim \lambda,[s([\lambda])]=[\lambda]$. Hence,

$$
[\lambda] \eta_{Q}^{(s)}([\lambda])(e)=[s([\lambda])](s([\lambda]) \backslash s([\lambda]))=[s([\lambda])]=[\lambda]=[\lambda] e .
$$

This completes the proof.

We set $\Delta_{1}=\left(\eta_{Q}^{(s)} \otimes 1_{Q}\right) \circ l_{Q}^{-1}, \Delta_{2}=\left(1_{Q} \otimes \eta_{Q}^{(s)}\right) \circ r_{Q}^{-1}$, and $\sigma_{i}=\Delta_{i} \circ m_{Q}(i=$ 1,2). It follows from Propositions $4,5,7$, and 8 that each $\left(Q, \sigma_{i}\right)(i=1,2)$ is a braided semigroup in Set $_{H}$ with the comultiplication $\Delta_{i}$, and a straightforward calculation shows that the morphisms $\sigma_{1}$ and $\sigma_{2}$ are exactly the same as those in (2.4). Theorem 2 thus proves Theorem 1.

REMARK 7. (1) Remarks 5 (3) and 6 (1) with Propositions 7 and 8 also induce Theorem 1.

(2) The construction [17] of dynamical Yang-Baxter maps using dynamical braces cannot produce the above dynamical Yang-Baxter map $\sigma_{1}$, if $\#(Q)>1$. Suppose, contrary to our claim, that there exists a dynamical brace that gives birth to $\sigma_{1}$ by the method in [17, Corollary 3.2.1]. Then this dynamical Yang-Baxter map satisfies the unitary condition $\sigma_{1}^{2}=1_{Q \otimes Q}$. As a result, $\sigma_{1}=\sigma_{1}^{2}=1_{Q \otimes Q}$ from Proposition 6. This contradicts the condition that $\#(Q)>1$.

Acknowledgment. The authors wish to express their thanks to Professor Kimio Ueno for his constant encouragement. The first and the second authors were supported in part by Grant-in-Aid for JSPS Fellows and KAKENHI (22540001), respectively.

\section{References}

[1] Adler, V. E., Bobenko, A. I. and Suris, Y. B., Geometry of Yang-Baxter maps: pencils of conics and quadrirational mappings, Comm. Anal. Geom. 12 (2004), no. 5, 967-1007. 
[ 2 ] BAxter, R. J., Partition function of the eight-vertex lattice model, Ann. Physics 70 (1972), 193-228; Exactly solved models in statistical mechanics, Academic Press, Inc., London, 1982.

[ 3 ] Bukhshtaber, V. M., Yang-Baxter mappings, Uspekhi Mat. Nauk 53 (1998), no. 6 (324), 241-242; translation in Russian Math. Surveys 53 (1998), no. 6, 1343-1345.

[ 4 ] DRInfel'D, V. G., Quantum groups, Proceedings of the International Congress of Mathematicians, Vol. 1, 2 (Berkeley, Calif., 1986), 798-820, Amer. Math. Soc., Providence, RI, 1987.

[ 5 ] DRINFEL'D, V. G., On some unsolved problems in quantum group theory, Quantum groups (Leningrad, 1990), 1-8, Lecture Notes in Math., 1510, Springer, Berlin, 1992.

[ 6 ] Etingof, P. and VArchenko, A., Solutions of the quantum dynamical Yang-Baxter equation and dynamical quantum groups, Comm. Math. Phys. 196 (1998), no. 3, 591-640.

[ 7 ] Etingof, P., Schedler, T. and Soloviev, A., Set-theoretical solutions to the quantum Yang-Baxter equation, Duke Math. J. 100 (1999), 169-209.

[ 8 ] Felder, G., Conformal field theory and integrable systems associated to elliptic curves, Proceedings of the International Congress of Mathematicians, Vol. 1, 2 (Zürich, 1994), 1247-1255, Birkhäuser, Basel, 1995.

[9] Gateva-Ivanova, T., A combinatorial approach to the set-theoretic solutions of the Yang-Baxter equation, J. Math. Phys. 45 (2004), 3828-3858.

[10] Gervais, J.-L. and Neveu, A., Novel triangle relation and absence of tachyons in Liouville string field theory, Nuclear Phys. B 238 (1984), no. 1, 125-141.

[11] Jimbo, M., A $q$-difference analogue of $U(\mathfrak{g})$ and the Yang-Baxter equation, Lett. Math. Phys. 10 (1985), no. 1, 63-69.

[12] KamiYa, N. and Shibukawa, Y., Dynamical Yang-Baxter maps associated with homogeneous pre-systems, J. Gen. Lie Theory Appl. 5 (2011), Article ID G110106.

[13] Kassel, C., Quantum groups, Graduate Texts in Mathematics, 155. Springer-Verlag, New York, 1995.

[14] Kouloukas, T. E. and Papageorgiou, V. G., 3D compatible ternary systems and Yang-Baxter maps, J. Phys. A 45 (2012), no. 34, 345204.

[15] MaC LANE, S., Categories for the working mathematician, Second edition. Graduate Texts in Mathematics, 5. Springer-Verlag, New York, 1998.

[16] MaJID, S., Foundations of quantum group theory, Cambridge University Press, Cambridge, 1995.

[17] Matsumoto, D. K., Dynamical braces and dynamical Yang-Baxter maps, J. Pure Appl. Algebra 217 (2013), no. 2, 195-206.

[18] Pflugfelder, H. O., Quasigroups and loops: introduction, Sigma Series in Pure Mathematics, 7. Heldermann Verlag, Berlin, 1990.

[19] RumP, W., A decomposition theorem for square-free unitary solutions of the quantum Yang-Baxter equation, Adv. Math. 193 (2005), 40-55.

[20] RumP, W., Braces, radical rings, and the quantum Yang-Baxter equation, J. Algebra 307 (2007), 153-170.

[21] ShibukawA, Y., Dynamical Yang-Baxter maps, Int. Math. Res. Not. 2005 (2005), 2199-2221.

[22] ShibukawA, Y., Dynamical Yang-Baxter maps with an invariance condition, Publ. Res. Inst. Math. Sci. 43 (2007), no. 4, 1157-1182.

[23] Shibukawa, Y., Survey on dynamical Yang-Baxter maps, Proceedings of noncommutative structures in mathematics and physics (Brussels, Belgium, 2008), 238-243, The Royal Flemish Academy of Belgium for Sciences and Arts, 2010.

[24] Shibukawa, Y., Dynamical braided monoids and dynamical Yang-Baxter maps, Quantum groups and quantum topology (edited by A. Masuoka), 80-89, RIMS Kôkyûroku 1714, 2010.

[25] Shibukawa, Y. and TAKeuchi, M., FRT construction for dynamical Yang-Baxter maps, J. Algebra 323 (2010), 1698-1728.

[26] Smith, J. D. H. and Romanowska, A. B., Post-modern algebra, Pure and Applied Mathematics (New York). A Wiley-Interscience Publication. John Wiley \& Sons, Inc., New York, 1999.

[27] TAKeUCHI, M., Matched pairs of groups and bismash products of Hopf algebras, Comm. Algebra 9 (1981), 
no. 8, 841-882.

[28] TAKeUCHI, M., Survey on matched pairs of groups-an elementary approach to the ESS-LYZ theory, Noncommutative geometry and quantum groups (Warsaw, 2001), 305-331, Banach Center Publ., 61, Polish Acad. Sci., Warsaw, 2003.

[29] Veselov, A. P., Yang-Baxter maps and integrable dynamics, Phys. Lett. A 314 (2003), no. 3, $214-221$.

[30] Woronowicz, S. L., Compact matrix pseudogroups, Comm. Math. Phys. 111 (1987), no. 4, 613-665.

[31] YANG, C. N., Some exact results for the many-body problem in one dimension with repulsive delta-function interaction, Phys. Rev. Lett. 19 (1967), 1312-1315; S matrix for the one-dimensional N-body problem with repulsive or attractive $\delta$-function interaction, Phys. Rev. 168 (1968), 1920-1923.

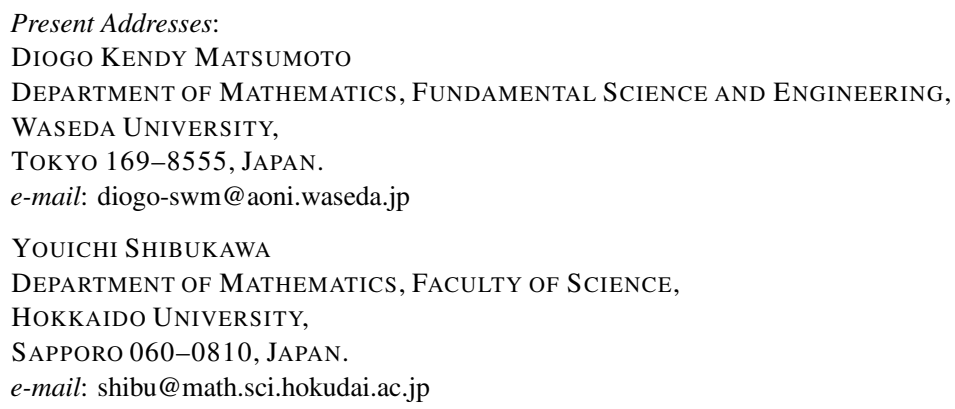

